\title{
Factores asociados a las demandas psiquiátricas a los servicios de urgencias prehospitalarios de Málaga (España)
}

\author{
José Guzmán-Parra, ${ }^{1,2,3}$ Ana Isabel Martínez-García, ${ }^{2,4}$ Cristobalina Guillén-Benítez, ${ }^{4}$ Serafina Castro-Zamudio, ${ }^{2,5}$ \\ Manolo Jiménez-Hernández, ${ }^{2,5}$ Berta Moreno-Küstner ${ }^{2,3,5}$
}

Artículo original

\section{ABSTRACT}

\section{Introduction}

In recent years, there has been a significant increase in the demand for prehospital emergency care in different countries.

\section{Objective}

The aim of the present study was to identify the variables associated with psychiatric calls to the Prehospital Emergency Care Services (PECS) in the province of Malaga.

\section{Method}

An observational retrospective study based on calls made to the PECS and registered in the computerized database of the Coordination Emergency Centre during one year $(N=163331)$. Independent variables included 1. sociodemographic variables: sex and age; and 2. variables related with the characteristics of each call: time of day, type of day, time of year, caller identification, number of resources needed, number of patients attended and type of solution. The $\chi^{2}$ test was used to compare of the variables. A multivariant logistic regression analysis was also carried out.

\section{Results}

Psychiatric calls accounted for $7 \%$ of the total calls and were associated with: younger age, female gender, calls made in the evenings and afternoons, a lower number of patients attended, the call being performed by other individual calling on the patient's behalf, and no ambulance transportation.

\section{Discussion and conclusion}

The calls concerned with mental health problems have specific characteristics which need to be taken into account in order to provide a better care for psychiatric patients.

Key words: Prehospital Emergency Care, mental health, utilization.

\section{RESUMEN}

\section{Introducción}

En los últimos años se ha producido un importante aumento de la demanda asistencial de urgencias a nivel prehospitalario.

\section{Objetivo}

El presente estudio tiene como objetivo principal identificar las variables asociadas a las demandas clasificadas como psiquiátricas a los Servicios de Urgencia y Emergencias Médicos Prehospitalarios (SUEMP) de la provincia de Málaga.

\section{Método}

Estudio observacional retrospectivo de las demandas registradas en la base de datos informatizada (computarizada) del Centro Coordinador de Urgencias y Emergencias durante un año $(N=163331)$. Se han considerado: 1) variables sociodemográficas: la edad y el sexo, y 2) variables relacionadas con la demanda: la franja horaria, el tipo de día, el trimestre del año, el sujeto alertante, el número de recursos movilizados, el número de personas atendidas y si hubo trasporte sanitario. Para la comparación de las variables se empleó la prueba $\chi^{2}$. También se realizó un análisis de regresión logística multivariante.

\section{Resultados}

El 7\% de las demandas a los SUEMP se clasificaron como psiquiátricas. Entre las variables relacionadas con las demandas psiquiátricas se encontraron tener menor edad, ser mujer, demanda realizada por la noche y la tarde, menor número de personas atendidas, que la alerta no fuera efectuada por el propio usuario y la no realización de traslado sanitario.

\section{Discusión y conclusión}

Las demandas por problemas de salud mental presentan características diferenciales al resto de demandas a los SUEMP, lo que hay que tener en cuenta para mejorar la atención a dichos pacientes.

Palabras clave: Servicios de emergencias prehospitalarios, salud mental, utilización de servicios.

Unidad de Gestión Clínica del Hospital Regional de Málaga.

2 Grupo Andaluz de Investigación Psicosocial (GAP) (CTS-945)

3 Instituto IBIMA. Málaga, España.

4 Unidad de Gestión Clínica de los Dispositivos de Cuidados Críticos y Urgencias del Distrito Sanitario Málaga-Coín Guadalhorce.

Departamento de Personalidad, Evaluación y Tratamiento Psicológico. Universidad de Málaga, España.

Correspondencia: Berta Moreno Küstner. Departamento de Personalidad, Evaluación y Tratamiento Psicológico. Universidad de Málaga. Campus de Teatinos s/n 29071 Málaga, España. Tel: 699 - 418971 . E-mail: berłamk@uma.es

Recibido primera versión: 16 de diciembre de 2015. Segunda versión: 6 de junio de 2016. Aceptado: 24 de octubre de 2016. 


\section{INTRODUCCIÓN}

En los últimos años se ha producido un importante aumento de la demanda asistencial de urgencias, tanto a nivel hospitalario ${ }^{1,2}$ como prehospitalario., Algunos estudios muestran que el mayor incremento se ha producido en las demandas de tipo psiquiátrico. ${ }^{4,5}$ Varios factores se han postulado como causantes de este aumento: ${ }^{6}$ descenso de camas psiquiátricas por habitante ocurrido en las décadas recientes, aumento del estrés psicosocial, incremento del consumo de alcohol entre la población joven, menor apoyo social y mayor aislamiento de la población; además, Larkin et al. ${ }^{7}$ hallaron que uno de cada tres pacientes con trastornos mentales que acudían a las urgencias hospitalarias lo hacía en ambulancia, por sólo uno de cada siete con otras patologías. Cuddeback et al. ${ }^{8}$ observaron que la mayoría de los traslados en ambulancia estaban asociados con el abuso de sustancias pero éstos eran los que menos quedaban ingresados en el hospital, siendo los trastornos del estado de ánimo y la esquizofrenia los que finalizaban más frecuentemente en ingreso. También se ha relacionado la hiperfrecuentación y el mal uso de los servicios de urgencias con los trastornos mentales, ${ }^{9}$ el abuso de sustancias ${ }^{10}$ y las dificultades socioeconómicas $^{11}$ y se han propuesto distintas iniciativas con el fin de mejorar esta situación. ${ }^{12-14}$

A pesar de que las personas con trastornos mentales utilizan de forma reiterada los Servicios de Urgencias y Emergencias Prehospitalarios (SUEMP) ${ }^{15}$ y del aumento de las demandas por problemas mentales, existen pocos estudios que analicen las demandas prehospitalarias por problemas mentales.

Los SUEMP se crearon en España en los años 1980 y principios de los 1990 con un doble objetivo: a) establecer un mecanismo de coordinación mediante un número de teléfono único de acceso (061) y b) crear dispositivos de emergencias públicos para la asistencia médica prehospitalaria y el traslado de pacientes a los centros sanitarios, por ejemplo, los Centros de Coordinación de Urgencias y Emergencias (CCUE) y los Dispositivos de Cuidados Críticos y Urgencias (DCCU). En España los SUEMP atienden aproximadamente a ocho millones de personas anualmente, ${ }^{16}$ suponiendo un coste importante para el sistema sanitario público.

En España, cada comunidad autónoma gestiona su propio sistema sanitario. La comunidad autónoma de Andalucía, con ocho millones de habitantes aproximadamente, cuenta con una red gestionada a través de la Empresa Pública de Emergencias Sanitarias (EPES). La coordinación de los diferentes dispositivos se realiza desde los CCUE situados en cada una de las ocho provincias andaluzas. De estos ocho centros, los de Sevilla y Málaga (este último es el lugar en el que se desarrolla el estudio) se consideran de alta complejidad, gestionando en la actualidad más de medio millón de demandas anuales.
El presente estudio tiene como objetivo principal comparar las demandas clasificadas como psiquiátricas con el resto de demandas a los SUEMP, así como identificar las variables asociadas a las demandas psiquiátricas.

\section{MÉTODO \\ Diseño y ámbito del estudio}

Se realizó un estudio transversal, observacional. Concretamente se revisó la información registrada durante el año 2008, en la base de datos informatizada del CCUE de Málaga. En este servicio se recogen todas las demandas de urgencias prehospitalarias realizadas en la provincia de Málaga, cuya población cubierta es de 1528851 habitantes aproximadamente.

En la provincia de Málaga los SUEMP trasladan pacientes a seis hospitales públicos, que ofrecen atención psiquiátrica en sendos servicios de urgencias hospitalarias. De estos seis hospitales, dos tienen unidades psiquiátricas de corta estancia para pacientes que requieren ingresos breves para su estabilización. Además existe un hospital psiquiátrico con gestión privada que cuenta con plazas concertadas con el sistema sanitario público en unidades de hospitalización psiquiátrica de corta, media y larga estancia.

La atención a los usuarios de los SUEMP se realiza en base a un sistema de triage utilizando el síntoma más importante o de más gravedad (síntoma guía) informado por el alertante (persona que realiza la llamada). Según el síntoma guía existe un protocolo de preguntas específico que realiza el profesional que contesta la llamada para determinar el nivel de prioridad: (1) emergencia, (2) urgencia no demorable, (3) urgencia demorable y (4) avisos domiciliarios; que genera una serie de acciones y recursos sanitarios a enviar. Es un objetivo de estos servicios el asignar un recurso (ambulancia o helicóptero) antes de tres minutos en las emergencias de prioridad 1. Los traslados sanitarios se realizan según el criterio médico del facultativo responsable de la demanda que valora la necesidad del mismo.

\section{Sujetos}

La base de datos analizada consta de un total de 321167 demandas. Para este estudio se eliminaron las demandas de tipo no sanitario que son aquellas que no implicaban la atención a personas con necesidades sanitarias $(n=74058)$ y, por otro lado, las que no fueron clasificadas en ninguna categoría por el CCUE ( $n=71977$ ), ya que la información no se ajustaba a ninguna de las categorías de dicho sistema de clasificación. Estas últimas demandas fueron eliminadas para evitar los sesgos que pudieran provocar, ya que la mayoría fueron demandas farmacológicas o inespecíficas. Además se realizó un control de calidad de la base de datos y se eliminaron los 
duplicados (coincidían el número identificativo, la fecha y la hora) y errores de registro (11 801). Por lo tanto, se han incluido todas aquellas demandas que provocaron un consejo sanitario (algún tipo de indicación por parte del CCUE sin necesidad de movilizar un recurso), la asignación de un recurso (algún tipo de atención sanitaria in situ) o una derivación a otro dispositivo sanitario (traslado a un hospital) y que estaban clasificados en alguna de las categorías específicas que utiliza el CCUE ( $n=163$ 331). Información más detallada del proceso de selección de la muestra aparece en la figura 1.

\section{Mediciones}

Se ha considerado como variable dependiente las demandas psiquiátricas. La clasificación de las demandas se basa en la información obtenida por teléfono por el CCUE, sobre los síntomas de mayor relevancia informados por el usuario al operador/a de la llamada, siendo el médico coordinador el responsable de la clasificación final de la demanda. Este sistema de clasificación es específico de los CCUE de Andalucía, y la mayoría de estos servicios en el resto de lugares utilizan un sistema de clasificación adaptado a las características locales. El sistema de clasificación del CCUE incluye 13 categorías principales (tabla 1) siendo una de ellas las demandas psiquiátricas, que constan a su vez de un total de siete subcategorías: amenaza suicida, incoherencia/cuadro confusional, nerviosismo, oposicio-

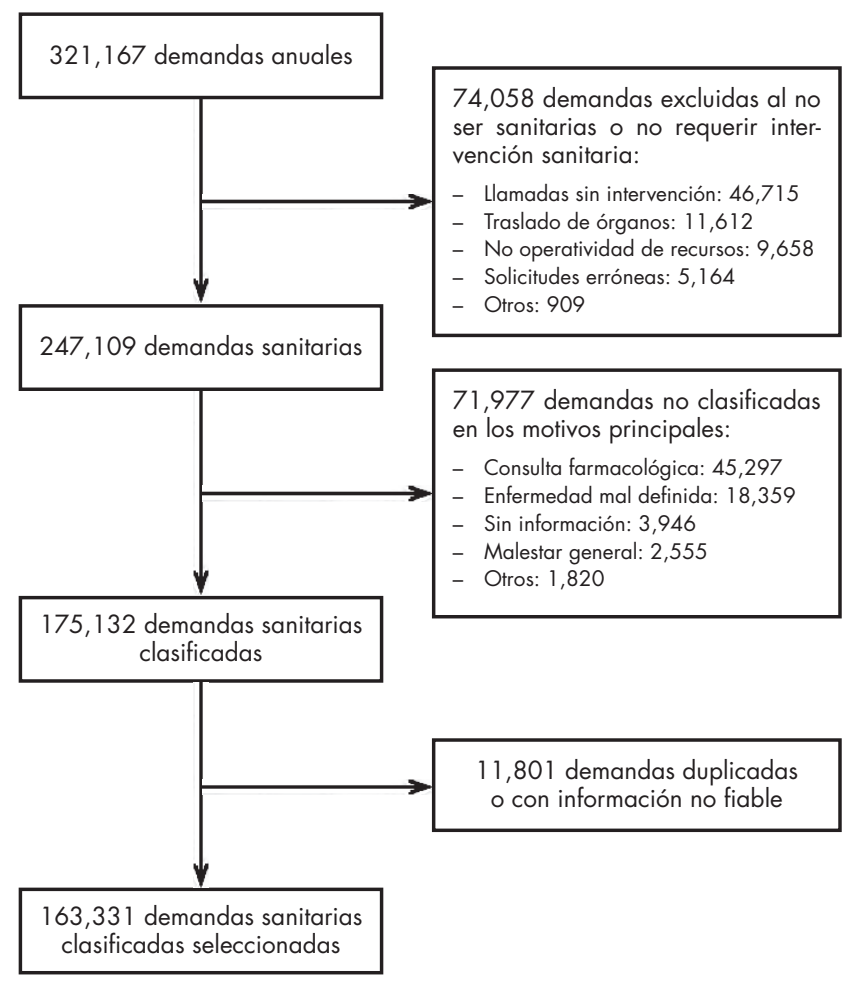

Figura 1. Diagrama de flujo del proceso de selección de la muestra de estudio. nismo, tristeza, violencia, otros y no clasificados (sin información suficiente). Dentro de las demandas psiquiátricas se añadieron para este estudio aquellas clasificadas como "ansiedad" incluidas en la categoría principal de "disnea". Dentro de la clasificación intoxicaciones/alergias existe un sub-apartado: intoxicaciones por tóxicos, drogas, medicamentos y alcohol. Puesto que dentro de este apartado pueden incluirse intoxicaciones por toma de medicación $\mathrm{u}$ otros tóxicos que no tienen que ver con el consumo de drogas y alcohol, se decidió no incluirlo dentro de las demandas psiquiátricas.

También se realizó un análisis de las características diferenciales entre los diferentes tipos de demandas psiquiátricas. Para ello se agruparon las demandas psiquiátricas en tres categorías: "amenaza suicida", siendo la conducta suicida uno de los problemas sociales más relevantes y poco estudiados en el ámbito de las emergencias;, "nerviosismo, ansiedad y tristeza", que tienen que ver con los cuadros de ansiedad y afectivos que son los más prevalentes en la población general, 18 y "otros".

Se han considerado como variables independientes, respecto a los usuarios: la edad, categorizada en intervalos (0-17, 18-44, 45-64, 65-79 y > 80 años; si bien para el modelo de regresión logística se utilizó la edad como variable continua) y el sexo; y respecto a las demandas se ha tenido en cuenta: la franja horaria (mañana [8:01 a 15:00], tarde [15:01 a 21:00] y noche [21:01 a 8:00]), el tipo de día (laborable o festivo) y el trimestre del año en que se realizó la demanda. También se ha analizado el sujeto alertante (usuario u otros); el número de recursos movilizados $(0,1$ y $>1)$, entendiendo por recurso movilizado la necesidad de la intervención de algún tipo de ambulancia o helicóptero (teniendo en cuenta que se pueden enviar varias ambulancias a una sola demanda) con o sin traslado a algún centro sanitario; el número de personas atendidas $(1,>1)$, y el tipo de resolución (traslado a centro sanitario, no traslado).

\section{Tabla 1}

Clasificación de los motivos de las demandas al Centro Coordinador de Urgencias y Emergencias de Málaga. $N=163331$

\begin{tabular}{lcc}
\hline Motivos de las demandas & $N$ & $\%$ \\
\hline Dolor no traumático & 34,984 & 21.4 \\
Alteraciones neurológicas y/o del nivel de la & 33,197 & 20.3 \\
conciencia & 17,496 & 10.5 \\
Disnea & 16,519 & 10.7 \\
Traumatismos & 15,987 & 9.8 \\
Alteración de las constantes vitales & 11,331 & 6.9 \\
Psiquiátricas & 9,116 & 5.6 \\
Accidentes de tráfico & 8,405 & 5.1 \\
Gastrointestinal & 5,133 & 3.1 \\
Demandas de enfermería & 4,697 & 2.9 \\
Intoxicaciones/alergias & 4,011 & 2.5 \\
Hemorragias & 2,133 & 1.3 \\
Ginecológicas/obstétricas/urinarias & 322 & 0.2 \\
Urgencias ambientales/agentes externos & & \\
\hline
\end{tabular}


Tabla 2

Comparación de las demandas psiquiátricas con las demandas no psiquiátricas: análisis bivariado y multivariado. $N=163331$

\begin{tabular}{|c|c|c|c|c|c|c|c|c|}
\hline \multirow[b]{2}{*}{ Características } & \multirow{2}{*}{$\frac{\text { Total }}{N}$} & \multicolumn{2}{|c|}{ Otras } & \multicolumn{2}{|c|}{ Psiquiátricas } & \multirow[b]{2}{*}{$\chi^{2}$} & \multicolumn{2}{|c|}{$\begin{array}{l}\text { Regresión logística } \\
(N=132416)\end{array}$} \\
\hline & & $N$ & $\%$ & $N$ & $\%$ & & ORa & IC 95\% \\
\hline Edad $^{a}$ & 152,395 & & & & & $6,964.0^{\dagger}$ & $0.97^{\dagger}$ & $0.972-0.974$ \\
\hline $0-17$ & & 4,312 & 3.04 & 292 & 2.70 & & & \\
\hline $18-29$ & & 10,136 & 7.20 & 1,647 & 15.30 & & & \\
\hline $30-44$ & & 16,470 & 11.60 & 3,676 & 34.20 & & & \\
\hline $45-59$ & & 17,958 & 12.70 & 1,931 & 18.00 & & & \\
\hline $60-75$ & & 31,893 & 22.50 & 1,252 & 11.60 & & & \\
\hline$>75$ & & 60,876 & 43.00 & 1,952 & 18.20 & & & \\
\hline Sexo & 150,300 & & & & & $181.0^{\dagger}$ & & $1.392-1.515$ \\
\hline Hombre & & 62,561 & 44.80 & 4,050 & 38.90 & & & \\
\hline Mujer & & 77,099 & 55.20 & 6,590 & 61.90 & & $1.45^{\dagger}$ & \\
\hline Franja horaria & 163,331 & & & & & $277.0^{\dagger}$ & & \\
\hline Mañana (8:01 a 15:00) & & 58,769 & 38.70 & 3,528 & 31.10 & & & \\
\hline Tarde (15:01 a $21: 00)$ & & 54,179 & 35.60 & 4,320 & 38.10 & & $1.26^{\dagger}$ & $1.196-1.319$ \\
\hline Noche (21:01 a 8:00) & & 39,052 & 25.70 & 3,483 & 30.70 & & $1.25^{\dagger}$ & $1.188-1.318$ \\
\hline Día de la semana & 163,331 & & & & & $13.0^{\dagger}$ & & \\
\hline Laborables & & 100,143 & 65.90 & 7,654 & 67.50 & & & \\
\hline Fin de semana & & 51,857 & 34.10 & 3,677 & 32.50 & & $0.91^{\dagger}$ & $0.871-0.950$ \\
\hline Trimestres & 163,331 & & & & & $46.0^{\dagger}$ & & \\
\hline Primero & & 35,116 & 23.10 & 2,356 & 20.80 & & & \\
\hline Segundo & & 35,857 & 23.60 & 2,762 & 24.40 & & $1.15^{\dagger}$ & $1.079-1.217$ \\
\hline Tercero & & 40,362 & 26.60 & 3,247 & 28.70 & & $1.18^{\dagger}$ & $1.114-1.252$ \\
\hline Cuarto & & 40,665 & 26.80 & 2,966 & 26.20 & & $1.12^{\dagger}$ & 1.059-1.191 \\
\hline$N^{\circ}$ recursos movilizados & 163,331 & & & & & $1642.0^{\dagger}$ & & \\
\hline 0 & & 32,530 & 21.40 & 4,281 & 37.80 & & & \\
\hline 1 & & 111,753 & 73.50 & 6,705 & 59.20 & & $0.58^{\dagger}$ & $0.550-0.607$ \\
\hline$>1$ & & 7,717 & 5.10 & 345 & 3.00 & & $0.41^{\dagger}$ & $0.360-0.463$ \\
\hline $\mathrm{N}^{\circ}$ personas atendidas & 153,711 & & & & & $4.7^{*}$ & & \\
\hline 1 & & 140,656 & 98.50 & 10,706 & 98.70 & & & \\
\hline$>1$ & & 2,210 & 1.50 & 139 & 1.30 & & $0.57^{\dagger}$ & $0.474-0.685$ \\
\hline Sujeto alertante & 143,524 & & & & & $6.0^{*}$ & & \\
\hline Otros & & 29,642 & 22.10 & 2,133 & 23.20 & & & \\
\hline Usuario & & 104,677 & 77.90 & 7,072 & 76.80 & & $0.84^{\dagger}$ & $0.792-0.885$ \\
\hline Traslado sanitario & 163,331 & & & & & $282.0^{\dagger}$ & & \\
\hline No & & 100,534 & 66.10 & 8,368 & 73.90 & & & \\
\hline Sí & & 51,466 & 33.90 & 2,963 & 26.10 & & $0.77^{\dagger}$ & $0.735-0.816$ \\
\hline
\end{tabular}

Nota: ORa: Odds Ratio ajustada; IC: Intervalo de Confianza; CCUE: Centro Coordinador de Urgencias y Emergencias;

a Para el modelo de regresión logística multivariante la edad se introdujo como variable continua y no por franjas de edad.

${ }^{*} p<0.05,{ }^{\dagger} p<0.001$

\section{Análisis estadísticos}

Para la comparación de las variables categóricas se empleó la prueba $\chi^{2}$. También se realizó un análisis de regresión logística multivariante tomando como variable dependiente las demandas de tipo psiquiátrico para conocer la asociación con las variables independientes. Se calcularon las Odds Ratios ajustadas (ORa) y sus intervalos de confianza (IC) al 95\% para cada categoría de las variables. Para analizar las características de las diferentes categorías de las demandas psiquiátricas se realizó un análisis bivariado mediante regresión logística multinomial. Todos los análisis estadísticos se hicieron utilizando el programa SPSS versión 15.0 para Windows.

\section{Consideraciones éticas}

El estudio fue aprobado por el Comité de Ética e Investigación Málaga Nordeste.

\section{RESULTADOS}

Se han analizado 163331 demandas a los SUEMP. De estas demandas, $33.3 \%$ requirieron traslado sanitario; de ellas, 
Tabla 3

Comparación de los diferentes tipos de demandas psiquiátricas: análisis bivariado

\begin{tabular}{|c|c|c|c|c|c|c|c|c|}
\hline \multirow[b]{2}{*}{ Características } & \multicolumn{2}{|c|}{$\begin{array}{l}\text { Nerviosismo, ansiedad } \\
\text { o tristeza (1) (referencia) }\end{array}$} & \multicolumn{2}{|c|}{$\begin{array}{c}\text { Amenazas } \\
\text { de suicidio (2) }\end{array}$} & \multicolumn{2}{|c|}{ Otras (3) } & \multicolumn{2}{|c|}{$\begin{array}{c}\text { Regresión } \\
\text { logísticamultinomial }\end{array}$} \\
\hline & $N$ & $\%$ & $N$ & $\%$ & $N$ & $\%$ & OR2 vs 1 & OR3 vs 1 \\
\hline $\operatorname{Edad}(N=10,750)^{a}$ & & & & & & & $0.978^{\dagger}$ & 1 \\
\hline $0-17$ & 182 & 2.8 & 29 & 2.8 & 81 & 2.5 & & \\
\hline $18-29$ & 941 & 14.6 & 211 & 20.1 & 495 & 15.2 & & \\
\hline $30-44$ & 2,119 & 32.9 & 444 & 42.3 & 1,113 & 34.1 & & \\
\hline $45-59$ & 1,133 & 17.6 & 235 & 22.4 & 563 & 17.2 & & \\
\hline $60-75$ & 805 & 12.2 & 92 & 8.8 & 355 & 10.9 & & \\
\hline$>75$ & 1,254 & 19.5 & 38 & 3.6 & 660 & 20.2 & & \\
\hline \multicolumn{9}{|l|}{$\operatorname{Sexo}(N=10,640)$} \\
\hline Hombre & 2,079 & 32.6 & 478 & 45.3 & 1,493 & 46.5 & & \\
\hline Mujer & 4,291 & 67.4 & 578 & 54.7 & 1,721 & 53.5 & $0.586^{\dagger}$ & $0.558^{\dagger}$ \\
\hline \multicolumn{9}{|l|}{ Franja horaria $(N=11,331)$} \\
\hline Mañana (8:01 a 15:00) & 2,040 & 30.3 & 330 & 28.2 & 1,158 & 33.7 & & \\
\hline Tarde (15:01 a $21: 00)$ & 2,574 & 38.3 & 456 & 38.9 & 1,290 & 37.5 & 1.128 & $0.826^{\dagger}$ \\
\hline Noche (21:01 a 8:00) & 2,109 & 31.4 & 385 & 32.9 & 989 & 28.8 & 1.095 & $0.883^{*}$ \\
\hline \multicolumn{9}{|c|}{ Día de la semana $(N=11,331)$} \\
\hline Laborables & 4,525 & 67.3 & 795 & 67.9 & 2,334 & 67.9 & & \\
\hline Fin de semana & 2,198 & 32.7 & 376 & 32.1 & 1,103 & 32.1 & 0.974 & 0.973 \\
\hline \multicolumn{9}{|l|}{ Trimestres $(N=11,331)$} \\
\hline Primero & 1,352 & 20.1 & 218 & 18.6 & 786 & 22.9 & & \\
\hline Segundo & 1,594 & 23.7 & 266 & 22.7 & 902 & 26.2 & 1.035 & 0.973 \\
\hline Tercero & 1,951 & 29.0 & 357 & 30.5 & 939 & 27.3 & 1.135 & $0.828^{\dagger}$ \\
\hline Cuarto & 1,826 & 27.2 & 330 & 28.2 & 810 & 23.6 & 1.122 & $0.763^{\dagger}$ \\
\hline \multicolumn{9}{|c|}{$N^{\circ}$ recursos movilizados $(N=11,331)$} \\
\hline 0 & 3,025 & 45.0 & 178 & 15.2 & 1,078 & 31.4 & & \\
\hline 1 & 3,542 & 52.7 & 933 & 79.7 & 2,230 & 64.9 & $6.536^{\dagger}$ & $2.320^{\dagger}$ \\
\hline$>1$ & 156 & 2.3 & 60 & 5.1 & 129 & 3.8 & $4.476^{\dagger}$ & $1.767^{\dagger}$ \\
\hline \multicolumn{9}{|c|}{$N^{\circ}$ personas atendidas $(N=10,845)$} \\
\hline 1 & 6,405 & 98.8 & 1060 & 99.0 & 3,241 & 98.5 & & \\
\hline$>1$ & 78 & 1.2 & 11 & 1.0 & 50 & 1.5 & 0.852 & 1.267 \\
\hline \multicolumn{9}{|l|}{ Sujeto alertante $(9,205)$} \\
\hline Otros & 1,283 & 23.1 & 204 & 26.2 & 646 & 22.5 & & \\
\hline Usuario & 4,276 & 76.9 & 574 & 73.8 & 2,222 & 77.5 & 0.844 & 1.032 \\
\hline \multicolumn{9}{|c|}{ Traslado sanitario $(N=11,331)$} \\
\hline No & 5,502 & 81.8 & 490 & 41.8 & 2,378 & 69.2 & & \\
\hline Sí & 1,221 & 18.2 & 681 & 58.2 & 1,059 & 30.8 & $6.223^{\dagger}$ & $2.007^{\dagger}$ \\
\hline
\end{tabular}

Notas: OR: Odds Ratio. El grupo de comparación de referencia en la regresión logística multinomial fue el de Nerviosismo, ansiedad o tristeza (1). 2 vs 1: Odds Ratio del grupo (2) en comparación con el grupo de referencia (1). 3 vs 1: Odds Ratio del grupo (3) en comparación con el grupo de referencia (1).

a Para el modelo de regresión logística multinomial la edad se introdujo como variable continua y no por franjas de edad.

${ }^{*} p<0,05,{ }^{\dagger} p<0,001$

$59.5 \%$ se realizó mediante ambulancia medicalizada, $22.9 \%$ con ambulancia básica sin equipo médico y $17.6 \%$ con otro tipo de recurso o no se recogió la información. De las que no presentaron traslado sanitario (66.7\%), en el $41.5 \%$ la asistencia se realizó en el domicilio, $21.0 \%$ se resolvieron mediante consejo sanitario por el operador/a, $7.8 \%$ fueron anuladas por el usuario y $29.7 \%$ tuvieron otro tipo de resolución. En cuanto a la prioridad: $14.4 \%$ se clasificó como emergencia, $61.9 \%$ como urgencia no demorable, $13.8 \%$ como urgencia demorable y $9.9 \%$ como avisos domiciliarios. La distribución de las demandas clasificadas por el CCUE en el año de estudio aparece en la tabla 1, siendo la más frecuente el dolor no traumático (21.4\%), seguida por las demandas por alteraciones neurológicas y/o nivel de la conciencia (20.3\%). El número de demandas psiquiátricas fue de 11331, situándose en el sexto lugar (6.9\%).

La comparación de las demandas psiquiátricas con las no psiquiátricas se muestra en la tabla 2. El $49.6 \%$ de las demandas psiquiátricas fueron para atención de personas entre 18 y 44 años. Sólo $2.7 \%$ fueron para personas menores de 18 años y 30\% fueron para personas mayores de 60 años. En lo referente al sexo, las demandas psiquiátricas estuvie- 
ron asociadas con el sexo femenino (61.9\%) con respecto a las no psiquiátricas (57.2\%; $p<0.001)$. Se produjeron en menor porcentaje por la mañana $(31.1 \%$ vs $38.7 \%)$, aumentando por la tarde $(38.1 \%$ vs $35.6 \%)$ y la noche $(30.8 \%$ vs $25.7 \%)(p<0.001)$. El porcentaje de demandas psiquiátricas en fin de semana fue menor en comparación al de las otras demandas y fueron más frecuentes en el $2^{\circ}$ y $3^{\circ}$ trimestre con respecto a las no psiquiátricas $(p<0.001)$. Se destaca que sólo el 3.0\% movilizó más de un recurso frente al 5.1\% de las demandas no psiquiátricas $(p<0.001)$. El número de personas atendidas fue significativamente menor en las demandas psiquiátricas $(p<0.05)$. En cuanto al sujeto alertante, en las demandas psiquiátricas fue menos probable que fuera el propio usuario $(76.8 \%)$ en comparación con las no psiquiátricas $(77.9 \% ; p<0.05)$. Las demandas psiquiátricas requirieron traslados sanitarios $(26.1 \%)$ en menor medida que las demandas de otro tipo (33.9\%; $p<0.001)$.

El modelo de regresión logística multivariante (tabla 2) explicó un $9.8 \%$ de la varianza máxima $\left(\mathrm{R}^{2}\right.$ de Nagelkerke $=$ 0.098). Las variables relacionadas con las demandas psiquiátricas (versus no psiquiátricas) fueron la edad ( $\mathrm{ORa}=0.97$; disminución de un 3\% de las demandas psiquiátricas por cada año de incremento de edad), el ser mujer (ORa $=1.45)$, demanda realizada por la noche $(\mathrm{ORa}=1.25)$ y la tarde $(\mathrm{ORa}=1.26)$, demanda realizada en día laborable (festivo vs laborable, $\mathrm{ORa}=0.91)$, demanda realizada en los últimos trimestres del año ( 2 vs $1, \mathrm{ORa}=1.15 ; 3$ vs $1, \mathrm{ORa}=1.18 ; 4$ vs $1, \mathrm{ORa}=1.12)$, demanda que no movilizó ningún recurso ( 1 vs $0, \mathrm{ORa}=0.58 ;>1$ vs $0, \mathrm{ORa}=0.41)$, haber atendido un menor número de personas (> 1 vs $1, \mathrm{ORa}=0.57$ ), alerta no efectuada por el usuario (usuario vs otros, ORa $=0.84$ ) y demanda que no requirió traslado sanitario (traslado vs no traslado, $\mathrm{ORa}=0.77$ ).

El porcentaje de los diferentes grupos dentro de las demandas psiquiátricas fue el siguiente: nerviosismo, ansiedad o tristeza (59.3\%), amenazas de suicidio (10.3\%) y otras $(30.4 \%)$. Las demandas categorizadas como amenazas de suicidio en comparación con las categorizadas como nerviosismo, ansiedad o tristeza se caracterizaron por una menor edad $(\mathrm{OR}=0.978)$, por estar menos asociadas al género femenino $(\mathrm{OR}=0.586$ ), por movilizar más recursos ( 1 vs 0 , $\mathrm{OR}=6.536 ;>1$ vs $0, \mathrm{OR}=4.476)$ y requerir más traslados sanitarios $(\mathrm{OR}=6.223)$. Las diferencias entre los tres grupos de demandas psiquiátricas aparecen resumidas en la tabla 3.

\section{DISCUSIÓN Y CONCLUSIÓN}

La principal conclusión de este estudio es que existe un perfil diferencial de las demandas psiquiátricas a los SUEMP de Málaga. Las características de las demandas psiquiátricas, comparadas con el resto, es que las primeras se realizan más frecuentemente por personas jóvenes, mujeres, por la noche $\mathrm{y}$ por la tarde, son realizadas en menor frecuencia por el propio usuario, representan un menor número de traslados a centros sanitarios y se atiende a un menor número de personas. Entre las fortalezas de este trabajo podemos destacar que se trata del primer estudio realizado en España, que se centra en las demandas de tipo psiquiátrico a los SUEMP y que se basa en todas las demandas registradas en un área concreta, a partir de la práctica clínica diaria, lo que representa la realidad asistencial de estos servicios. ${ }^{19}$ Además, el análisis de datos prehospitalarios se puede considerar como una fuente de información importante para la vigilancia de la salud pública. ${ }^{4}$

En este estudio las demandas de tipo psiquiátrico suponen aproximadamente el $7 \%$, cifra ligeramente superior a la encontrada en otras investigaciones de nuestro entorno. En España, según Pacheco et al. ${ }^{20}$ el 3.7\% de las demandas fueron clasificadas según la CIE-9-MC (Clasificación Internacional de Enfermedades, $9^{\mathrm{a}}$ revisión) como grupo V: psiquiátricas, mientras que Fuentes Lema y López Pérez $^{19}$ ofrecieron una cifra que no llegaba al 1\%. Munjal et al., ${ }^{4}$ estudiando las demandas prehospitalarias en un área urbana de la ciudad de Nueva York, refieren que el 5.5\% presentaban problemas psiquiátricos, concluyendo que el mayor incremento de las demandas a las urgencias prehospitalarias estaba relacionado con las categorías problemas psiquiátricos/uso de sustancias (+ 5.6\%/año). En Madrid, las demandas de tipo psiquiátrico supusieron un $10.8 \%$ y un $12.0 \%$ en 2001 y en $2002,{ }^{5}$ lo que se aproxima a los resultados de este estudio. Una de las explicaciones sobre la discrepancia entre las cifras de demandas por problemas psiquiátricos puede ser debido a las diferencias en las clasificaciones de las demandas utilizadas en los diferentes lugares.

Respecto al género, el porcentaje de mujeres con demandas de tipo psiquiátrico es mayor (61.9\%) que las que lo hicieron por otro tipo de problemas. Esto es lo que cabría esperar dada la mayor morbilidad psiquiátrica en el sexo femenino. Sin embargo, en muchos estudios los servicios de urgencias hospitalarios son más frecuentados por hombres los cuales presentan mayor prevalencia de trastornos de personalidad y consumo de tóxicos. ${ }^{21}$

En cuanto a la edad, las personas que realizaron demandas de tipo psiquiátrico se concentran en la franja que va desde los 18 a los 44 años, frente al resto de demandas que fueron más frecuentes a partir de los 65 años. Este hecho posiblemente esté relacionado con la naturaleza de las problemáticas de salud mental, cuya edad de inicio suele estar comprendida en una franja de edad joven, mientras que una gran proporción de enfermedades de tipo físico suelen comenzar a una edad más avanzada. Con respecto al horario, las demandas psiquiátricas se produjeron en mayor medida por la tarde y la noche lo que podría estar relacionado con la búsqueda de atención rápida de algunos pacientes con problemas mentales y una mayor dificultad de acceso a los servicios especializados de salud mental en estos horarios. 
Cabe destacar que las demandas psiquiátricas fueron más frecuentes en los meses de verano a diferencia del resto que lo fueron principalmente en invierno. Una posible explicación a este hecho podría estar en que, como sugieren Ortega et al., ${ }^{22}$ hay factores relacionados con los flujos de demanda asistencial, como podría ser la dificultad de acceder a los dispositivos asistenciales de salud mental, o el incremento de la población de veraneantes en una ciudad como Málaga, que durante su estancia podría presentar algún tipo de alteración psicopatológica y que no puede acceder a los servicios especializados de forma inmediata. Nuestros resultados son coherentes con otro estudio que señala un mayor uso de las urgencias hospitalarias por pacientes psiquiátricos en los días de más calor y humedad. ${ }^{23}$

Es más probable, así mismo, que las demandas psiquiátricas no movilicen ningún recurso y presenten menos traslados sanitarios, lo que podría relacionarse con el hecho de que algunos profesionales de los servicios de urgencias prehospitalarios subestiman la importancia de las demandas psiquiátricas. ${ }^{7,24}$

También, por los resultados del estudio se deduce que hay características diferenciales dentro de los grupos de demandas psiquiátricas, siendo las demandas relacionadas con el suicido las que requieren más recursos y son realizadas por una población más joven, coincidiendo estos datos con otros estudios realizados en España. ${ }^{25}$

Entre las limitaciones del estudio cabe indicar que el hecho de utilizar datos secundarios, obtenidos de una base de datos clínica puede comprometer la fiabilidad de algunos registros, no obstante, como se ha comentado en la sección de métodos, se ha llevado a cabo una intensa depuración y revisión de los datos para minimizar los posibles errores y dada la muestra tan amplia consideramos que su impacto es limitado. Otra limitación es el gran porcentaje de demandas incluidas en el apartado "no clasificadas" $\mathrm{y}$, por lo tanto, no analizadas en este estudio, lo que impide conocer exactamente todos los motivos de las demandas a estos servicios y que pueden constituir un sesgo en los resultados, aunque se repitieron los análisis incluyendo estas demandas junto a las demandas no psiquiátricas y los resultados fueron similares, por lo que no creemos que supongan un sesgo de importancia. Otro aspecto a destacar es que algunas cifras de odds ratios, aunque son estadísticamente significativas debido a la amplia muestra, son pequeñas, por lo que su significación clínica es limitada. Por otro lado, el que la mayoría de los sistemas de emergencias prehospitalarios no usen un sistema de clasificación internacional también limita las comparaciones con otros servicios de otros países y otras comunidades autónomas españolas.

Por último, podemos señalar que este trabajo puede ayudar al dimensionamiento de las urgencias prehospitalarias en general y las demandas por problemas de salud mental en particular. También nos ha permitido analizar la variabilidad en la práctica clínica y a establecer y monitorizar objetivos asistenciales. Además hemos podido identificar las características diferenciales de las demandas urgentes de personas con problemas de salud mental, las cuales representan un problema de salud pública por la gran repercusión que tiene este colectivo en la población en general y por la gravedad de los síntomas.

Como recomendación futura, planteamos que el estudio en profundidad de las necesidades asistenciales y urgentes que presenta un colectivo tan vulnerable como son las personas con enfermedad mental, nos ayudaría a mejorar la atención sanitaria a este grupo de pacientes.

\section{Financiamiento}

Junta de Andalucía (PI/08/332; CTS-945)

\section{Conflicto de intereses}

Los autores declaran no tener ningún conflicto de intereses.

\section{Agradecimientos}

Los autores quieren agradecer al Distrito Sanitario Málaga, particularmente a Maximiliano Vilaseca y a Javier Navarro por su apoyo logístico, así como a David Doblas por su colaboración en esta línea de investigación.

\section{REFERENCIAS}

1. Ovens HJ, Chan BT. Heavy users of emergency services: a population-based review. CMAJ. 2001;165(8):1049-1050.

2. Tankel AS, Di Palma MJ, Kramer KM, van der Zwan R. Increasing impact of mental health presentations on New South Wales public hospital emergency departments 1999-2006. Emerg Med Australas. 2011;23(6):689-696.

3. Cano del Pozo MI, Rabanaque Hernández MJ, Feja Solana C, et al. Estudio de la frecuentación de un servicio de urgencias extrahospitalario. Emergencias. 2008;20(3):179-186.

4. Munjal KG, Silverman RA, Freese J, et al. Utilization of emergency medical services in a large urban area: description of call types and temporal trends. Prehosp Emerg Care. 2011;15(3):371-380.

5. Román MI, de Miguel AG, Garrido PC, et al. Epidemiologic intervention framework of a prehospital emergency medical service. Prehosp Emerg Care. 2005;9(3):344-354.

6. Pajonk FG, Schmitt P, Biedler A, et al. Psychiatric emergencies in prehospital emergency medical systems: a prospective comparison of two urban settings. Gen Hosp Psychiatry. 2008;30(4):360-366.

7. Larkin GL, Claassen CA, Pelletier AJ, Camargo CA. National study of ambulance transports to United States emergency departments: importance of mental health problems. Prehosp Disaster Med. 2006;21(2):82-90.

8. Cuddeback G, Patterson PD, Moore CG, Brice JH. Utilization of emergency medical transports and hospital admissions among persons with behavioral health conditions. Psychiatr Serv. 2010;61(4):412-415.

9. Scott J, Strickland AP, Warner K, Dawson P. Describing and predicting frequent callers to an ambulance service: analysis of 1 year call data. Emerg Med J. 2013:1-7.

10. Knowlton A, Weir BW, Hughes BS, et al. Patient demographic and health factors associated with frequent use of emergency medical services in a midsized city. Acad Emerg Med. 2013;20(11):1101-1111.

11. Tangherlini N, Pletcher MJ, Covec MA, Brown JF. Frequent use of emergency medical services by the elderly: a case-control study using paramedic records. Prehosp Disaster Med. 25(3):258-264. 
12. Naya Cendón C, Rodríguez de la Iglesia MA, Blanco Rodríguez E. Estudio de la frecuentación a un servicio de urgencias extrahospitalario: motivos de consulta, opinión de los usuarios y posibles soluciones. Cad atención primaria. 2010;17(1):15-18.

13. Weiss SJ, Ernst AA, Ong M, et al. Effect of a social services intervention among 911 repeat users. Am J Emerg Med. 2005;23(4):492-496.

14. Rinke ML, Dietrich E, Kodeck T, Westcoat K. Operation care: a pilot case management intervention for frequent emergency medical system users. Am J Emerg Med. 2012;30(2):352-357.

15. Bulbena A, Martin LM, Arcega JM. Experiencia de una Unidad Móvil de Urgencias Psiquiátricas en Barcelona (EMSE-061). Equipo Multidisciplinar de Soporte Especializado. Av en Salud Ment Relac. 2007;6:1-14.

16. Pacheco Rodríguez A, Burusco S, Senosiáin Larrión MV. Prevalencia de procesos y patologías atendidos por los servicios de emergencia médica extrahospitalaria en España. An Sist Sanit Navar. 2010;33(1):37-46.

17. Larkin GL, Beautrais AL. Emergency departments are underutilized sites for suicide prevention. Crisis. 2010;31(1):1-6.

18. Haro JM, Palacin C, Vilagut G, et al. Prevalencia y factores asociados de los trastornos mentales en España: resultados del estudio ESEMeD-España. Med Clínica. 2006;126(12):445-451.
19. Fuentes Lema M, Lopez Pérez A. Análisis de las urgencias extrahospitalarias en la comarca de Verín. Cad atención Primaria. 2008;15:29-32.

20. Pacheco A, Burusco S, Senosiáin MV. Prevalencia de los procesos y patologías atendidos por los servicios de emergencia médica extrahospitalaria en España. An Sist Sanit Navar. 2010;33 Suppl 1:37-46.

21. Usall i Rodié J, Vázquez-Barquero JL, Montero I, et al. Género y salud mental en un mundo cambiante. Gac Sanit. 2004;18(1):175-181.

22. Ortega Maján MT, Rabanaque Hernández MJ, Júdez Legaristi D, Cano del Pozo MI, Abad Díez JM, Moliner Lahoz J. Perfil de los usuarios y motivos de demanda del Servicio de Urgencias extrahospitalario 061. Emergencias. 2008;20(1):27-34.

23. Vida S, Durocher M, Ouarda TB, Gosselin P. Relationship between ambient temperature and humidity and visits to mental health emergency departments in Québec. Psychiatr Serv. 2012;63(11):1150-1153.

24. Schmidt TA, Atcheson R, Federiuk C, et al. Hospital follow-up of patients categorized as not needing an ambulance using a set of emergency medical technician protocols. Prehosp Emerg Care. 5(4):366-370.

25. Mejías Y, García Caro MP, Schmidt J, Quero A, Gorlat B. Estudio preliminar de las características del intento de suicidio en la provincia de Granada. An Sist Sanit Navar. 2011;34:431-441. 